\title{
EXAMPLES OF NATURAL EXTENSIONS OF NONSINGULAR ENDOMORPHISMS
}

\author{
K. G. DAJANI AND J. M. HAWKINS
}

(Communicated by Palle E. T. Jorgensen)

\begin{abstract}
We extend a nonsingular noninvertible map $T$ on $(X, \mathscr{B}, \mu)$ to a minimal nonsingular automorphism containing $T$ as a factor and preserving some measure theoretic properties of $T$. We call the automorphism the natural extension of $T$. We compute some examples of natural extensions: first when $T$ is a shift with $\mu$ a product measure, and then generalize the result to some nonexact examples as well.
\end{abstract}

\section{INTRODUCTION AND BACKGROUND}

We have shown in an earlier paper that the maximal automorphic factor of an endomorphism carries measure theoretic information about the endomorphism [1]. A related invertible map is the minimal natural extension which has been studied in [8-10] for example. We give some examples to illustrate the differences and connections between the two automorphisms. In particular, the role of recurrent vs. nonrecurrent measures is irrelevant in the tail automorphism factor studied in [1]. We will show here that the opposite is true for the natural extension; this is also discussed in [8]. The examples in this paper first appeared as the last section of an unpublished preprint by the authors circulated in 1990; the rest of the preprint comprises [1,2]. We have generalized some of the original results here.

We begin with the definition of the natural extension of a nonsingular endomorphism. Throughout this paper we assume we are given a standard Borel space $(X, \mathscr{B}, \mu)$ with $\mu$ a $\sigma$-finite measure on $\mathscr{B}$ and an endomorphism $T$ which satisfies the following conditions.

(1) $T$ is forward and backward nonsingular (for all $A \in \mathscr{B}, T A, T^{-1} A \in$ $\mathscr{B}$, and $\left.\mu(A)=0 \Leftrightarrow \mu(T A)=0 \Leftrightarrow \mu\left(T^{-1} A\right)=0\right)$.

(2) $T$ is countable-to-one (for $\mu$ a.e. $x \in X$, the set $\left\{T^{-1} x\right\}$ is at most countable).

(3) $T$ is ergodic (for all $A \in \mathscr{B}$ with $T^{-1} A=A$, either $\mu(A)=0$ or $\mu(X \backslash A)=0)$.

Received by the editors July 30, 1992.

1991 Mathematics Subject Classification. Primary 28D05.

Key words and phrases. Ergodic theory, nonsingular endomorphisms.

The authors received partial support from National Science Foundation grant DMS-9103656. 
(4) $T$ is conservative (for each $A \in \mathscr{B}$, there exists $m \in \mathbb{N}$ such that $\left.\mu\left(A \cap T^{-m} A\right)>0\right)$.

By $\omega_{\mu}$ we denote the unique $T^{-1} \mathscr{B}$ measurable function satisfying: for every $f \in L^{1}(X, \mathscr{B}, \mu)$,

$$
\int_{X} f(T x) \cdot \omega_{\mu}(x) d \mu(x)=\int_{X} f(x) d \mu(x) .
$$

Clearly $\omega_{\mu}(x) \equiv\left(d \mu / d \mu T^{-1}\right)(T x)$, and we call $\omega_{\mu}$ the Radon-Nikodym derivative of $T$ with respect to $\mu$. Any function $\omega$ satisfying (*) for all $L^{1}$ functions is called a Markovian for $T$ and $\mu$. Given any measure $\nu=h d \mu$, the function $\omega_{\nu} \cdot h /(h \circ T)$ is a Markovian with respect to $T$ and $\mu$ but is not in general equal to $\omega_{\mu}$. We have $\omega_{\nu}=\left((h \circ T) / E_{\mu}\left(h \mid T^{-1} \mathscr{B}\right)\right) \cdot \omega_{\mu}$ a.e. where $E_{\mu}(\cdot \mid \mathscr{A})$ denotes the usual conditional expectation onto the sub- $\sigma$-algebra $\mathscr{A} \subseteq \mathscr{B}$ in $(X, \mu)$ [5]; conversely, every Markovian $\omega$ gives rise to an equivalent measure $\nu=\omega d \mu$ with $\omega_{\nu}=\omega \circ T$. From the above, we have that changing from $\mu$ to an equivalent measure does not guarantee a cohomologous Radon-Nikodým derivative (one with $\left.\omega_{\nu}=((h \circ T) / h) \cdot \omega_{\mu}\right)$; when we write $\omega_{\nu} \sim \omega_{\mu}$ we will mean that (the measures $\nu$ and $\mu$ are equivalent and) the Markovians are cohomologous. We recall that, for invertible $T$, the notions of equivalent measures and cohomologous Radon-Nikodým derivatives are identical.

Definition 1.1. We say that the endomorphism $T$ of $(X, \mathscr{B}, \mu)$ is a factor map of the automorphism $S$ of $(W, \mathscr{S}, \gamma)$ if there exists a map $\pi:(W, \mathscr{S}, \gamma) \rightarrow$ $(X, \mathscr{B}, \mu)$ such that:

(1) $A \in \mathscr{B} \Leftrightarrow \pi^{-1} A \in \mathscr{S}$, and $\mu(A)=0 \Leftrightarrow \gamma\left(\pi^{-1} A\right)=0$; and

(2) $\pi \circ S=T \circ \pi \quad \gamma$ a.e.

Definition 1.2. An automorphism $S$ of $(W, \mathscr{S}, \gamma)$ is said to be a natural extension of $T$ on $(X, \mathscr{B}, \mu)$ if there exists a factor map $\pi:(W, \mathscr{S}, \gamma) \rightarrow$ $(X, \mathscr{B}, \mu)$ satisfying:

(1) $\mathscr{S}=\bigvee_{n=0}^{\infty} S^{n}\left(\pi^{-1} \mathscr{B}\right)(\gamma \bmod 0)$, and

(2) $\omega_{\gamma} \sim \omega_{\mu} \circ \pi$; equivalently, there exists a measure $\gamma^{\prime} \sim \gamma$ such that $\omega_{\gamma^{\prime}}=\omega_{\mu} \circ \pi$.

In papers [8] and [10] it is shown that natural extensions are unique in the following sense.

Proposition 1.3. If $T_{1}$ on $\left(X_{1}, \mathscr{B}_{1}, \mu_{1}\right)$ is isomorphic to $T_{2}$ on $\left(X_{2}, \mathscr{B}_{2}, \mu_{2}\right)$ via an isomorphism $\phi$ and if $\omega_{\mu_{1}} \sim \omega_{\mu_{2} \phi}$, then their natural extensions are isomorphic [10].

Definition 1.4. We say the measure $\mu$ of the endomorphism $T$ is recurrent if there exists an $f \in L^{1}(X, \mathscr{B}, \mu)$ such that $\sum_{i=0}^{\infty}\left(f\left(T^{i} x\right) \cdot \prod_{k=0}^{i-1} \omega_{\mu}\left(T^{k} x\right)\right)=\infty$ for $\mu$ a.e. $x \in X$.

A Markovian $\omega$ is recurrent if the measure associated to it is recurrent (cf. [5] or [9]).

We will give examples and results in this paper to illustrate that equivalent but noncohomologous measures have qualitatively different natural extensions. Also, two nonisomorphic endomorphisms can give rise to isomorphic natural 
extensions. These examples illustrate that there is still something lacking in a canonical method for choosing a natural extension for a non-measure-preserving conservative endomorphism.

\section{ONE-SIDED NONSINGULAR BERNOULLI SHIFTS}

We review briefly some results about one-sided shift spaces. In this section we assume that $X=\prod_{i=0}^{\infty}\{0,1, \ldots, n-1\}_{i}$ is the one-sided shift space on $n$ states, $T$ is the shift map, and $\mu=\prod_{i=0}^{\infty} \mu_{i}$ with $\mu_{i}(\{j\})>0$ for all $j \in$ $\{0, \ldots, n-1\}$. In addition, we assume $\mu$ is nonsingular for the shift. By applying various results, we have the following.

Proposition 2.1 [4, 5, 7]. Any one-sided nonsingular shift with nonatomic product measure is exact and hence ergodic.

Proof. It is enough to show that a nonsingular shift measure is ergodic for the odometer by [5]. This follows from the conditions for nonsingularity for the shift [7] and ergodicity conditions for the odometer [4].

We construct two nonisomorphic finite measure-preserving one-sided Bernoulli shifts with isomorphic natural extensions. Using the same method we show how, for a fixed Bernoulli shift, changing to equivalent measures can give rise to uncountably many nonisomorphic nonsingular natural extensions. Our technique is to work with a nonrecurrent measure equivalent to an invariant one in each case; for each we obtain a nonsingular invertible map satisfying Definition 1.2. The natural extensions we obtain with these measures are isomorphic copies of the dissipative two-sided shift given by Krengel in [12] and Hamachi in [3]. The one-sided shifts we construct are isomorphic to the $\left(\frac{1}{2}, \frac{1}{2}\right)$ i.i.d. Bernoulli shift on two states and the $(1 /(1+\lambda), \lambda /(1+\lambda))$ i.i.d. Bernoulli shift respectively, with $\lambda \in(0,1)$. It is well known that the natural extensions of these shifts with respect to the i.i.d. measures are the two-sided versions of the same measures. Clearly choosing these measures gives nonisomorphic natural extensions. This contrasts with Proposition 2.13 of [1] regarding the automorphic factor, where changing to an equivalent measure does not affect the resulting invertible map but gives the same map and a cohomologous factor measure. The fact that nonrecurrent measures give dissipative natural extensions follows from Definition 1.2(2). Examples of two nonisomorphic endomorphisms (one Markov, one Bernoulli) with the same natural extension have been given earlier [11].

The examples are constructed as shifts on the measure space $X=\prod_{i=0}^{\infty}\{0,1\}_{i}$ with the product $\sigma$-algebra of Borel sets which we will denote by $\mathscr{B}$. The first measure that we put on $X$ is the product measure $\mu=\prod_{i=0}^{\infty} \mu_{i}$ defined as follows. We fix any $\lambda \in(0,1)$ and define $\mu_{0}(\{0\})=1 /(1+\lambda)$ and $\mu_{0}(\{1\})=\lambda /(1+\lambda)$. Then for all $i \geq 1$ we define $\mu_{i}(\{0\})=\frac{1}{2}=\mu_{i}(\{1\})$. The transformation $T$ is the one-sided shift. Clearly $T$ is two-to-one everywhere.

We write $X=C_{0} \cup C_{1}$, where $C_{j}=\left\{x: x_{0}=j\right\}$. Using results of Kakutani [7] we compute that $\theta_{\mu T}$ is constant on $C_{j}$ : for all $x \in C_{0}$,

$$
\theta_{\mu T}(x)=\theta_{\mu T}\left(x_{0}\right)=\frac{d \mu \circ T^{-1}}{d \mu}(x)=\frac{\lambda+1}{2},
$$


and for all $x \in C_{1}$,

$$
\theta_{\mu T}(x)=\frac{d \mu \circ T^{-1}}{d \mu}(x)=\frac{\lambda+1}{2 \lambda} .
$$

Using this plus the identity $\theta_{\mu T}(x)=\sum_{y \in T^{-1}(x)} 1 / J_{\mu T}(y)$, we have that the Jacobian is constant on cylinders of length $\geq 2$, and for $x \in C_{j k}=\left\{x: x_{0}=\right.$ $\left.j, x_{1}=k\right\}$ with $j, k \in\{0,1\}$, we have

$$
J_{\mu T}(x)=\frac{\mu_{0}(k)}{\mu_{0}(j) \mu_{1}(k)}=\frac{2 \mu_{0}(k)}{\mu_{0}(j)} .
$$

Since $\omega_{\mu}(x)=(1) /\left(\theta_{\mu T} \circ T\right)$, for all $x \in C_{j k}$ we have that $\omega_{\mu}(x)=\mu_{0}(k) / \mu_{1}(k)$ $=2 \mu_{0}(k)$. From this one computes that, for any $i \geq 1, \omega_{\mu}(i, x)=$ $(2 /(1+\lambda))^{i} \lambda^{p(x)}$ where $p(x)=p\left(x_{1}, \ldots, x_{i}\right)=x_{1}+\cdots+x_{i}$; clearly this function is constant on cylinders of length $i+1$. The nonsingularity of $T$ follows from the proof of Theorem 1 in [3], and estimates given in the same proof also show that $\mu$ is a nonrecurrent measure for $T$.

The natural extension of $T$ with respect to $\mu$ is the two-sided shift with the following product measure (which is the dissipative shift studied by Krengel in [12] and Hamachi in [3]). Denoting the natural extension by $T^{\prime}$ on the measure space $\left(X^{\prime}, \mathscr{B}^{\prime}, \mu^{\prime}\right)$, we have that $X^{\prime}=\prod_{i=-\infty}^{\infty}\{0,1\}_{i}$ and $\mu^{\prime}=\prod_{i=-\infty}^{\infty} \mu_{i}^{\prime}$ with $\mu_{i}^{\prime}=\mu_{i}$ for $i \geq 0$, and for $i<0, \mu_{i}^{\prime}=\mu_{0}$, i.e., $\mu_{i}^{\prime}(\{0\})=1 /(1+\lambda)$ and $\mu_{i}^{\prime}(\{1\})=\lambda /(1+\lambda)$. One can verify that $\omega_{\mu^{\prime} T^{\prime}}=\omega_{\mu T} \circ \pi \mu^{\prime}$ a.e. where $\pi: X^{\prime} \rightarrow X$ is the natural projection.

We now give a transfer function which takes $\mu$ to an equivalent recurrent and invariant probability measure $\bar{\mu}$. We define $h(x)=h\left(x_{0}\right)=(\lambda+1) / 2$ if $x_{0}=0$ and $h(x)=h\left(x_{0}\right)=(\lambda+1) / 2 \lambda$ if $x_{0}=1$; in other words, $h(x)=$ $\theta_{\mu T}(x)$. Letting $\bar{\mu}=h d \mu$, we now compute $\omega_{\bar{\mu}}(x)$. We denote by $E_{\mu}^{1}(h)$ the conditional expectation of $h$ onto $T^{-1} \mathscr{B}$; i.e., $E_{\mu}^{1}(h)=E_{\mu}\left(h \mid T^{-1} \mathscr{B}\right)$. By [5],

$$
\omega_{\bar{\mu}}(x)=\frac{h \circ T}{E_{\mu}^{1}(h)}(x) \cdot \omega_{\mu}(x)=\frac{1}{E_{\mu}^{1}(h)}(x) .
$$

However, from the above formulas one easily computes that $E_{\mu}^{1}(h)=1$ since $E_{\mu}^{1}(h)(x)=\omega_{\mu}(x) \cdot \sum_{y \in T^{-1}(T x)} h(y) / J_{\mu T}(y)$. It is easy to see that $\bar{\mu}$ is in fact the infinite product measure each of whose factor measures is the $\left(\frac{1}{2}, \frac{1}{2}\right)$ measure; that is, $T$ on $(X, \bar{\mu})$ is actually the $\left(\frac{1}{2}, \frac{1}{2}\right)$ Bernoulli shift. The original measure $\mu$ is an equivalent nonrecurrent measure. Letting $\lambda$ range over all positive reals, we obtain uncountably many equivalent nonrecurrent measures all of whose natural extension measures are mutually singular. (This follows from Kakutani's conditions [7].)

The second measure that we put on $X$ is the related product measure $\nu=$ $\prod_{i=0}^{\infty} \nu_{i}$ defined as follows. Using the same $\lambda \in(0,1)$ as above, we define $\nu_{i}(\{0\})=1 /(1+\lambda)$ and $\nu_{i}(\{1\})=\lambda /(1+\lambda)$ for all $i \geq 1$ and set $\nu_{0}(\{0\})=$ $\frac{1}{2}=\nu_{0}(\{1\})$. The transformation $S$ is still the one-sided shift on $(X, \nu)$. A computation similar to that above shows that $\nu$ is equivalent to the i.i.d. product measure $\bar{\nu}$ with $\bar{\nu}_{i}(\{0\})=1 /(1+\lambda)$ and $\bar{\nu}_{i}(\{1\})=\lambda /(1+\lambda)$ for every factor measure. It is easy to check that the natural extension for $S$ on $(X, \nu)$ is isomorphic to the natural extension for $T$ on $(X, \mu)$, even though the extensions for $S$ on $(X, \bar{\nu})$ and $T$ on $(X, \bar{\mu})$ are not isomorphic.

This leads to the general result about one-sided shifts with product measure. 
Theorem 2.2. Let $X=\prod_{i=0}^{\infty}\{0,1, \ldots, n-1\}_{i}$ with the product $\sigma$-algebra of Borel sets $\mathscr{B}$, and consider the product measure $\mu=\prod_{i=0}^{\infty} \mu_{i}$ which is nonsingular for the one-sided shift $T$. Then the natural extension of $T$ is the two-sided shift $T^{\prime}$ on $X^{\prime}=\prod_{i=-\infty}^{\infty}\{0,1, \ldots, n-1\}_{i}$, with measure $\mu^{\prime}=\prod_{i=-\infty}^{\infty} \mu_{i}$ where $\mu_{i}=\mu_{0}$ for all $i<0 . T^{\prime}$ is conservative if and only if $\mu$ is a recurrent measure for $T$.

Theorem 2.3. Assume $T$ on $(X, \mathscr{B}, \mu)$ is as in Theorem 2.2, and suppose that there is some $M$ such that $\mu_{i}=\mu_{j} \equiv \mu_{M}$ for all $i, j>M$. Then $\mu$ is recurrent and invariant under $T^{k}$ for all $k$ iff $\mu_{i}=\mu_{M}$ for all $i \in \mathbb{N}$.

Proof. $(\Leftrightarrow)$ Clearly $T$ is an i.i.d. Bernoulli shift under this hypothesis.

( $\Rightarrow$ ) If $\mu_{i} \neq \mu_{M}$ for some $i<M$, then $\mu$ is not recurrent with respect to $T^{i}$ using the same arguments given in the example above.

\section{NATURAL EXTENSIONS OF SOME NONEXACT ENDOMORPHISMS}

In $[1,2]$ we construct a two-to-one endomorphism $T$ on the product space

$$
X=Y \times Y^{+} \equiv \prod_{i=-\infty}^{\infty}\{0,1\}_{i} \times \prod_{j=0}^{\infty}\{0,1\}_{j}
$$

and a Borel measure $\mu$ on $X$ with the following properties: (1) $T$ is the product of the two-sided shift with the one-sided shift; (2) $T$ is nonsingular, conservative, and ergodic with respect to $\mu$; (3) $T$ admits no $\sigma$-finite invariant measure equivalent to $\mu$; and (4) $T$ is neither exact with respect to $\mu$, nor is $\mu$ the product measure of an exact with an automorphic measure. We describe briefly the construction.

We denote by $\mathscr{B} \times \mathscr{B}^{+}$the product Borel $\sigma$-algebra on $Y \times Y^{+}$. We define the measure $\nu$ on $(Y, \mathscr{B})$ to be the type III two-sided Bernoulli shift measure constructed by Hamachi in [3]. Then the measure on $Y \times Y^{+}$will be of the form $\mu(C)=\int_{Y} \rho_{y}\left(C \cap \alpha^{-1} y\right) d \nu(y)$, where $\alpha$ is the projection map onto the first factor of $Y \times Y^{+}$. We specify the measures $\rho_{y}$ on $\left(Y^{+}, \mathscr{B}^{+}\right)$according to the following algorithm. We fix any $\lambda \in(0,1)$. We define two measures $\rho^{0}$ and $\rho^{1}$ on the space $\{0,1\}$ by $\rho^{0}(0)=\rho^{0}(1)=\frac{1}{2}$ and $\rho^{1}(0)=1 /(1+\lambda), \rho^{1}(1)=$ $\lambda /(1+\lambda)$. For each $y \in Y$, we define $\rho_{y}$ to be the infinite product measure given by $\rho_{y}=\prod_{i=0}^{\infty} \rho^{y_{i}}$. That is, we consider $y=\left(\ldots, y_{-1}, y_{0}, y_{1}, \ldots, y_{n}, \ldots\right)$ and the $i$ th factor in the measure $\rho_{y}$ is $\rho^{j}$ if and only if $y_{i}=j$. Each $\rho_{y}$ will be an infinite product of factors of two different measures on $\mathrm{Y}^{+}$and, by results of Kakutani [7], for $\nu$ a.e. $y \in Y, \rho_{y}$ will be singular with respect to the shift $\sigma$.

We denote the invertible shift on $Y$ by $\Phi$; then $(\Phi y)_{i}=y_{i+1}$. It follows that $\rho_{\Phi y}=\prod_{i=0}^{\infty} \rho^{y_{i+1}}=\prod_{i=1}^{\infty} \rho^{y_{i}}$. The proofs of properties (1)-(3) are given in [2]. With respect to the structure given above, we prove the following.

Proposition 3.1. On the product space

$$
W=Y \times Z \equiv \prod_{i=-\infty}^{\infty}\{0,1\}_{i} \times \prod_{j=-\infty}^{\infty}\{0,1\}_{j}
$$

with the Borel sets $\mathscr{B} \times \mathscr{B}$, the product shift map $\Phi \times \sigma(y, z)=(\Phi y, \sigma z)$ with the measure $\gamma(C)=\int_{Y} \rho_{y}\left(C \cap \alpha^{-1} y\right) d \nu(y)$ with $\rho_{y}=\prod_{i=-\infty}^{\infty} \rho^{y_{i}}$ is the natural extension of $T$. 
Proof. Clearly the first condition of Definition 1.2 is satisfied. By [2, Proposition 4.12] since

$$
d\left(\rho_{\Phi^{-1} y} \sigma^{-1}\right)(z) / d \rho_{y}=1 \quad \gamma \text { a.e. }
$$

it follows that $\omega_{\gamma}(y, z)=\omega_{\nu}(y) \quad \gamma$ a.e.

We note that the structure of the maximal automorphic factor plays no role in the method used in computing the natural extension in Proposition 3.1. In particular, we can consider the following general construction.

Given a Lebesgue space $(Y, \mathscr{F}, \nu)$ with $\Phi$ a conservative, nonsingular, ergodic automorphism of $Y$. Consider $Z=\prod_{i=0}^{\infty}\{0,1, \ldots, n-1\}_{i}$ with the product $\sigma$-algebra of Borel sets $\mathscr{D}$, and let $\sigma$ denote the shift as a point transformation on $(Z, \mathscr{D})$. Given any family $\left\{\rho_{y}\right\}_{y \in Y}$ of measures on $(Z, \mathscr{D})$ satisfying for $\nu$ a.e. $y \in Y$ :

(1) for any $B \in \mathscr{D}$, the map $y \rightarrow \rho_{y}(B)$ is measurable from $Y$ to $\mathbb{R}$;

(2) $\rho_{y}=\prod_{i=0}^{\infty} \rho_{y}^{(i)}$ with $0<\rho_{y}^{(i)}(\{j\})<1$ for each $j \in\{0, \ldots, n-1\}$;

(3) $\rho_{\Phi y}=\rho_{y} \sigma^{-1}$; equivalently, $\rho_{\Phi y}^{(i)}=\rho_{y}^{(i+1)}$.

We form the product space $(Y \times Z, \mathscr{F} \times \mathscr{D}, \mu)$ as in 3.1, defining

$$
\mu(A \times B)=\int_{A} \rho_{y}(B) d \nu(y) \text { for } A \in \mathscr{F}, B \in \mathscr{D} .
$$

By [2] it follows that $\mu$ is conservative, nonsingular, and ergodic for the $n$-toone endomorphism $T=\Phi \times \sigma$. Let $T^{\prime}$ denote the natural extension of $T$, $Z^{\prime}=\prod_{i=-\infty}^{\infty}\{0,1, \ldots, n-1\}_{i}$ with $\mathscr{D}^{\prime}$ its Borel $\sigma$-algebra.

Theorem 3.2. $T^{\prime}=\Phi \times \sigma$ on $X^{\prime}=Y \times Z^{\prime}$ with measure $\mu^{\prime}(A \times B)=$ $\int_{A} \mu_{y}(B) d \nu(y)$ for each $A \in \mathscr{F}, B \in \mathscr{D}^{\prime}$. Each $\mu_{y}$ is a product measure defined by $\mu_{y}^{(i)}=\rho_{y}^{(i)}, i \geq 0$, and $\mu_{y}^{(i)}=\rho_{\Phi^{i} y}^{(0)}, i<0$.

\section{REFERENCES}

1. K. Dajani and J. Hawkins, Quotient relations and automorphic factors of countable-to-one maps, preprint, 1991.

2. _ Rohlin factors, product factors, and joinings for $n$-to-one endomorphisms, Indiana Univ. Math. J. 42 (1993), 237-258.

3. T. Hamachi, On a Bernoulli shift with non-identical factor measures, Ergodic Theory Dynamical Systems 1 (1981), 273-284.

4. T. Hamachi and M. Osikawa, Ergodic groups of automorphisms and Krieger's theorems, Sem. Math. Sci., vol. 3, Keio Univ., Yokohama, 1981.

5. J. Hawkins, Amenable relations for endomorphisms, Trans. Amer. Math. Soc. (to appear).

6. J. Hawkins and C. Silva, Noninvertible transformations admitting no absolutely continuous $\sigma$-finite invariant measure, Proc. Amer. Math. Soc. 11 (1991), 455-463.

7. S. Kakutani, On equivalence of infinite product measures, Ann. of Math. (2) 49 (1948), 214-224.

8. C. Silva, On $\mu$-recurrent nonsingular endomorphisms, Israel J. Math. 61 (1988), 1-13.

9. C. Silva and P. Thieullen, The subadditive ergodic theorem and recurrence properties of Markovian transformations, J. Math. Anal. Appl. 154 (1991), 83-99.

10. _ A skew product entropy for nonsingular endomorphisms, preprint, 1991. 
11. P. Walters, Some results on the classification of non-invertible measure preserving transformations, Lecture Notes in Math., vol. 318, Springer-Verlag, New York, 1973, pp. 266-276.

12. U. Krengel, Transformations without finite invariant measure have finite strong generators, Lecture Notes in Math., vol. 160, Springer-Verlag, New York, 1970, pp. 133-157.

Department of Mathematics, University of South Alabama, Mobile, Alabama 36688

Current address: Fac. der Technische Wiskunde en Informatica (SSOR), Mekelweg 4, 2628 CD Delft, The Netherlands

E-mail address: karma@dutiosa.twi.tudelft.nl

Department of Mathematics, University of North Carolina at Chapel Hill, Chapel Hill, NorTh Carolina 27599

E-mail address: jmh@math.unc.edu 